\title{
Surgical management and outcome of spinal alveolar soft part sarcoma (ASPA): a case series of five patients and literature review
}

Chenglong Zhao ${ }^{1 *+}$, Xin Gao ${ }^{1 \dagger}$, Jian Yang ${ }^{1 \dagger}$, Zhenxi $\mathrm{Li}^{1}$, Xiaopan Cai ${ }^{1}$, Tao Tan $^{1}$, Tianhui Hou ${ }^{1,2}$, Wangjun Yan ${ }^{1}$, Xinghai Yang ${ }^{1}$, Cheng Yang ${ }^{1 *}$, Tielong Liu ${ }^{1 *}$ and Jianru Xiao ${ }^{1 *}$

\begin{abstract}
Background: Alveolar soft part sarcoma (ASPS) is a rare entity of soft tissue malignancies with uncommon spinal involvements. Surgical management should be the best choice of cure.

Methods: Five patients with spinal ASPS were interviewed retrospectively, where data was collected. The relevant literatures were also systematically examined. Thereafter, patient and surgical data were obtained and pooled for prognostic analysis.

Results: A total of five patients with eight surgeries were reviewed retrospectively, and three patients previously reported were also included. All patients were surgically treated, where five of them underwent additional adjuvant therapies such as chemotherapy, radiotherapy, and targeted therapy in order to manage their local and/or systematic diseases. One patient was lost in follow-up. For the remaining seven patients, the mean follow-up period was $19.7 \pm 8$. 8 months, two succumbed to disease while five were alive at the time of the study.

Conclusions: Surgical management is shown to be the most important and the most effective treatment strategy for spinal ASPS, whereas adjuvant therapies made little impact. The prognostic factors for spinal ASPS are primary or metastatic lesions, neurological status, disease progression, systematic conditions, and resection approaches.
\end{abstract}

Keywords: Spine, ASPS, Surgical management, Prognostic factors

\section{Background}

Alveolar soft part sarcoma (ASPS), a rare entity of soft tissue malignancies, predominantly affects young adolescents and accounts for approximately $0.4-1.0 \%$ of all soft tissue sarcomas [1, 2]. ASPS is first described by Christopherson et al [3] in 1952 as a unique kind of tumors with uncertain histogenesis. It is characterized by pseudoalveolar, or organoid, arrangement of cells in

\footnotetext{
*Correspondence: 18501646030@163.com; ddyc2001@163.com; czyylt|@163.com; xiaojianruvip@163.com

Chenglong Zhao, Xin Gao and Jian Yang are co-first author. Cheng Yang, Tielong Liu and Jianru Xiao are co-corresponding authors

${ }^{\dagger}$ Equal contributors

'Spine Tumor Center, Department of orthopedic oncology, Changzheng Hospital, Second Military Medical University, Huangpu District, Fengyang Rd 415\#, Shanghai, China

Full list of author information is available at the end of the article
}

relation to numerous delicate endothelial-lined vascular channels and septa. ASPS is still classified as "tumors of uncertain differentiation" by the WHO today [4].

ASPS is a tumor with relatively indolent growth pattern, which commonly originates from the muscles and deep soft tissues of the trunk and the extremities; however, metastases may be present at the time of presentation or occur decades after the primary tumor resection [5-8]. The most effective treatment for ASPS is surgical excision while en-bloc resection offers the best chance of cure. Nevertheless, ASPS was previously reported to be refractory to chemotherapy and radiotherapy [9]. Both primary and metastatic spinal ASPS are rarely reported in the literature. There are only sporadic case reports published, which focus on the diagnosis and management [9-12]. In this case series, a retrospective review was performed to 
report the preliminary experiences of the clinical features and treatment strategies of spinal ASPS, the current literatures are also systematically reviewed.

\section{Methods}

\section{Patients review}

This report retrospectively reviewed patients that were surgically treated and confirmed as spinal ASPS postoperatively in our institution from January 2005 to December 2014. Patient and surgical data such as general information (age and sex), radiological findings, pre- and post-operative status, treatment strategies, operation details, complications, and pathological findings were collected. Lesion classification were classified as primary, locally recurred (surgically treated before), or metastatic. Other organ involvements and metastasis status were also documented. Operation details including the time of surgery, intraoperative blood loss, and reconstruction strategies were recorded. Treatment strategies include surgical management and adjuvant therapies such as chemotherapy, radiotherapy, and targeted therapy. The preoperative and 1-month postoperative Frankel score were calculated and compared for each patient. All patients were followed up after the surgery (every 3 months in the first year and 6 months thereafter), and the progression of their diseases were documented (local recurrence, metastasis, or none). At the last followup, the condition of each patient was confirmed by telephone calls and classified as no evidence of disease (NED), alive with disease (AWD), and died of disease (DOD). Permission from the hospital ethics committee and written informed consents from all patients were obtained before the beginning of the study.

\section{Literature review}

The sporadic case reports on spinal ASPS are treasured as valuable resources because the disease type being discussed and explored here is extremely rare with a very low morbidity. A PubMed search was conducted with the combination of the "alveolar soft part sarcoma and spine" since 2000 using the advanced search builder. Three articles were found and all included in the current study. Two independent researchers performed extended literature analysis fulfilling the requirement of this retrospective review. Detailed data were obtained and were pooled together with our clinical data; subsequent prognostic analyses were also performed.

\section{Results}

\section{General description}

Five patients underwent a total of eight surgeries were reviewed retrospectively, and three patients previously reported in the literatures were also included (Table 1). These eight patients have a mean age of $28.4 \pm 8.7$ years, with a female to male ratio of $5: 3$. There is a total of four sacrum and seven mobile spine lesions, symptoms include pain, numbness and weakness of the extremities, and/or palpable mass, which were later surgically resected. Other metastatic sites include the lung, the scull, and the pubis. The primary site of tumors was the sacrum for four patients, the thoracic spine for one patient, and the leg for one patient (the primary site of patient no. 8 was not available).

\section{Treatment details}

All patients were surgically treated, where five of them also underwent adjuvant therapies such as chemotherapy, radiotherapy, and targeted therapy to manage their local and/or systematic diseases. All patients suffered from neurological defects due to cord and/or radical compression, and the majority number of patients had their symptoms alleviated postoperatively. Nine piecemeal and two en bloc surgeries were performed. The time of surgery and intraoperative blood loss varies, with an average of $301.1 \pm 247.8 \mathrm{~min}(110-880)$ and $2162.5 \pm$ $1395.9 \mathrm{ml}$ (1000-5000), respectively. Reconstructions were performed for all patients postoperatively except for patient no. 2 who underwent low sacrectomy.

Two patients suffered from postoperative complications. Patient no.5 was a 16-year-old girl, who developed wound disunion and necrosis after the first surgery. After conservative treatment for 20 days, she underwent a second surgical procedure where her wound healed 15 days thereafter. Moreover, one patient reported by Lizzati et al. suffered from meningocele after the en bloc resection but made a full recovery after 40 days of hospitalization.

\section{Follow-up}

The mean follow-up time was $19.7 \pm 8.8$ months (8-34). Two patients had tumor local recurrence postoperatively and underwent further surgical procedures. Patient no.4 was alive with disease 21 months after the second surgery, and patient no. 5 succumbed to the disease 4 months after her third surgery (Fig. 1). The poor outcome of this 16year-old girl might be owing to local disease recurrence and the early onset of distant metastasis. The rest three patients were still alive at the time of the follow-up, where two of them had no evidence of disease. The two patients underwent en bloc resections and were reported earlier had different outcomes. One died 26 months postoperatively while the other one was alive with no evidence of disease after 18 months follow-up.

\section{Discussion}

ASPS is a malignant tumor, which accounts only 0.4$1 \%$ of all soft tissue sarcomas, with a higher incidence amongst patients between 15-35 years of age, but rare before age 5 and after age 50 years $[1,2,13,14]$. 


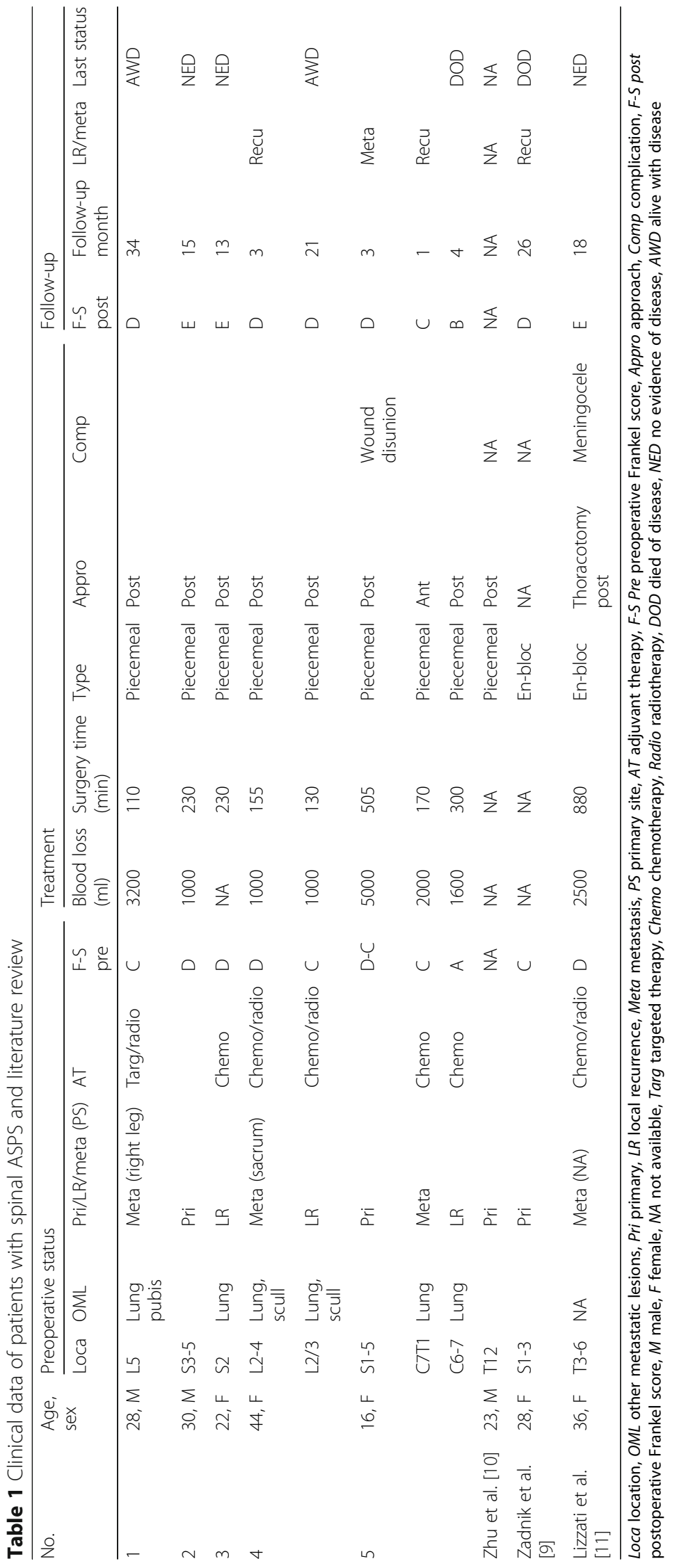




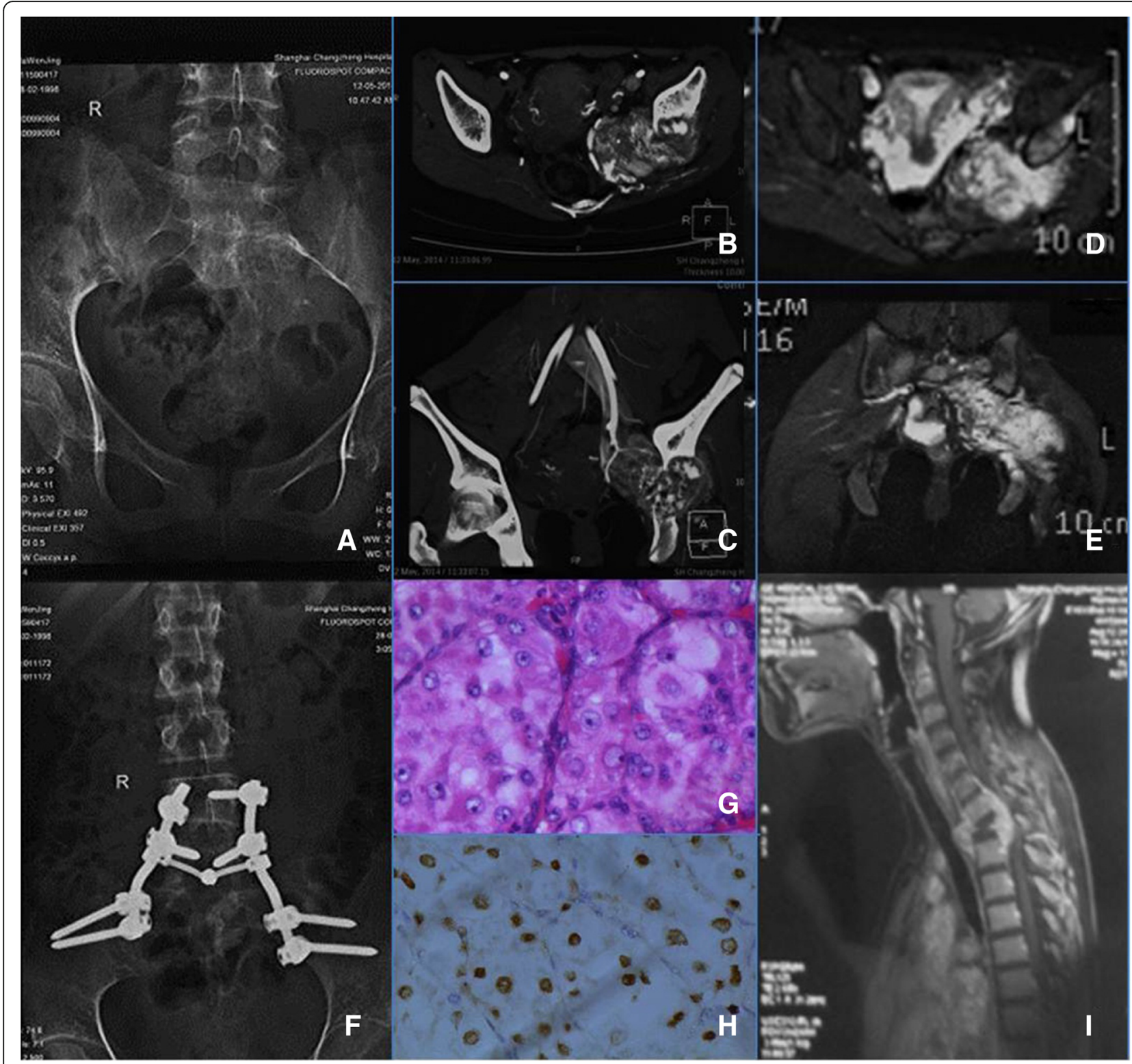

Fig. 1 Patient no. 5 suffered from pain in the buttock and gait abnormality for 2 months, X-ray (a), CT (b, c), and MRI (d, e) were performed preoperatively and a large tumor in the sacrum was revealed. A fine needle biopsy confirmed ASPS where surgical resection was indicated. After the tumor resection, screw and rod reconstruction was performed (f). The post operative histological examination (g) and the TFE3 positive immunochemistry (h) confirmed the pathology diagnosis of ASPS. Metastasis was detected 3 months later (i) and two further surgeries were performed. Unfortunately, this patient succumbed to the disease 5 months after the first surgery

The disease has obvious female predominance in the first three decades and reverses thereafter, the reported female to male ratio is approximately $2: 1$ and it is well reflected in this case series [13]. Although spinal involvements were extremely rare for ASPS, both primary and metastatic ASPS that erode the spinal column were represented in this report. In metastatic ASPS, more lesions were found in the mobile spines, whereas primary ASPS was more commonly seen in the sacrum.
The symptoms of spinal ASPS lack specificity. It may resemble neurological defects which initially present as pain and numbness of the innervated areas, followed by loss of mobility and loss of sensory receptions; furthermore, in the worst cases, this may progress into paralysis as seen in this case series as well as published literatures. Some patients also developed a soft palpable mass at the paraspinal areas of the buttocks. The radiological features of spinal ASPS are consistent with lesions at other locations but with characteristic osseous destruction plus 
a paraspinal soft tissue mass $[8,15]$. Magnetic resonance (MR) images may assist in the diagnosis and differential diagnosis of this relatively rare disease by a combination of heterogeneous high signal on both T1- and T2weighted images, with or without multiple intra- or extra-tumoral signal voids [16].

Pathological examinations, including histological and genetic analyses, have been treated as the gold standard diagnostic criteria and have an increased number of applications recently $[1,17,18]$. While Christopherson et al firstly reported and named ASPS as a distinctive and unrecognized soft tissue tumor, the histological features of ASPS were later described by pathologists [19-21]. The most important feature of ASPS is the characteristic ASPL/TFE3 fusion recognized using immunohistochemistry examinations, which is considered of great diagnostic value [22].

Surgical management indeed offer the best prognosis and should be considered the most important and the most effective treatment strategy for spinal ASPS as previously suggested [11]. At early stages, patients are asymptomatic; however, upon diagnosis, there have always been neurological defects and large size tumors identified. Surgical resections can directly remove the majority or the entirety of the tumor mass, which quickly lead to spinal cord or radicular decompression. This can alleviate suffering, improve the quality of life, or even prolong life expectancy. Although en bloc resection have been widely accepted and considered to be the best choice for soft tissue sarcomas, including ASPS, but it was not always achievable $[9,12]$, this is usually due to the large tumor size and the complexity of the local anatomical structures. Spinal ASPS is always accompanied with a large soft tissue mass, which might infiltrate into the soft paraspinal spaces and/or erode the surrounding vital nerve roots and vessels. Whether these structures can be sacrificed should be cautiously considered based on the general condition of the patient, which includes systematic disease progression, potential prognoses, and the preference of the patient. Although en bloc resection may prolong survival, the sacrifice of certain vital structures may compromise the quality of life for patients. All five patients in this review and one from the literature underwent piecemeal resection, whereas the other two from the literature underwent resection using an en bloc fashion. The duration of surgery and intraoperative blood loss ranges, from our experience, the spinal ASPS is rich in the vessels and it was challenging to deal with the intraoperative blood loss. Preoperative embolization of the tumor vessels might be helpful but no conclusion could be drawn yet. These features highlighted the difficulties in the surgical management of spinal ASPS.

Unfortunately, the value of adjuvant therapies for ASPS was limited. Four of our patients received adjuvant therapies. In three cases, this may have helped to control the systematic disease, but no impact was seen on the spinal lesions. In fact, chemotherapy and radiotherapy are more commonly considered as palliative strategies for hospice care [23, 24]. A number of ongoing clinical trials are focusing on the targeted therapies of ASPS, but no conclusions can be drawn yet [25-28].

Currently, the recognized prognostic factors of ASPS are age, tumor size, and metastases status, whereas the histological feature is thought to have no prognostic significance $[1,5,29,30]$. Three patients of our cases together with one from literature had metastatic spinal ASPS and were all successfully treated. The prognoses of these patients can be optimistic. However, the primary spinal ASPS may indicate a poor prognosis seen from both of our cases and the literature. Two of the patients with primary ASPS died while the other one (no. 3) had a local recurrence. Furthermore, the poor prognosis of patient no. 5 may be a combination of young age, large tumor size, rapid lesion progression, poor neurological status, early multi-organ metastasis, ineligibility to en bloc resection, and lack of effective adjuvant therapies. From our preliminary clinical experience and knowledge, it can be concluded that the poor prognostic factors for spinal ASPS may include primary spinal lesions, severe neurological defects, rapid lesion progression with early metastasis, and ineligibility to en bloc resection; on the other hand, patients with metastatic spinal lesions and well-controlled systematic disease may have a good prognosis. However, this conclusion requires further investigation using larger sample size due to the limitation of small patient number in the current study.

\section{Conclusions}

In summary, this report has presented a case series of five spinal ASPS patients and systematically reviewed related literatures. It is concluded that surgical management is the most important and the most effective treatment strategy for spinal ASPS while adjuvant therapies had little effects. The prognostic factors for spinal ASPS include primary or metastatic lesions, neurological defects, disease progression, systematic conditions, and resection approaches. The limitation of this study is the small patient number; however, due to the rare nature of this tumor type, clinical trials with large number of patients and randomized designs may require multiinstitutional cooperation.

\section{Abbreviations}

ASPS: Alveolar soft part sarcoma; AWD: Alive with disease; DOD: Died of disease; MR: Magnetic resonance; NED: No evidence of disease

\section{Acknowledgements}

We express our sincere thanks to Dr. Song Gao from Deakin University, School of Medicine, Melbourne, Australia, for her kind help in corrections of languages of this manuscript. 


\section{Funding}

This project is supported by the Science and Technology Commission of Shanghai Municipality. Award number 14140901202 to Dr. Cheng Yang.

\section{Availability of data and materials}

The patient data will not be shared. All of the patient data were collected from clinical notes of the Changzheng Hospital. All patients provided written consent for the storage of their information in the hospital database only.

\section{Authors' contributions}

$C Z$ and $Z L$ conceived the study. TL and JX designed the study. $T T, T H, W Y$, and $X Y$ performed the clinical data collection and extraction. $C Z, C Y$, and $X C$ performed the systematic review and data extraction. $C Z, X G$, and $J Y$ performed the statistical analysis. $C Z$ and $Z L$ drafted and revised the manuscript. CY helped in the revision of the manuscript after original review of the editorial board and offered the funding assistant. TL and JX gave the final approval of the revision. All authors read and approved the final manuscript.

\section{Competing interests}

The authors declare that they have no competing interests.

\section{Consent for publication}

Not applicable.

\section{Ethics approval and consent to participate}

The study was approved by the Research Ethics Board (REB) of the Changzheng Hospital. All patients provided written consents for the storage of their information in the hospital database and for the use of this information in this research.

\section{Author details}

'Spine Tumor Center, Department of orthopedic oncology, Changzheng Hospital, Second Military Medical University, Huangpu District, Fengyang Rd 415\#, Shanghai, China. ${ }^{2}$ College of Physical Education and Health, East China Normal University, Minhang District, Dongchuan Rd 500\#, Shanghai, China.

Received: 16 July 2016 Accepted: 1 February 2017

\section{Published online: 06 February 2017}

\section{References}

1. Folpe AL, Deyrup AT. Alveolar soft-part sarcoma: a review and update. J Clin Pathol. 2006;59:1127-32

2. Sandberg A, Bridge J. Updates on the cytogenetics and molecular genetics of bone and soft tissue tumors: alveolar soft part sarcoma. Cancer Genet Cytogenet. 2002;136:1-9.

3. Christopherson WM, Foote Jr FW, Stewart FW. Alveolar soft-part sarcomas. Structurally characteristic tumors of uncertain histogenesis. Cancer. 1952;5: 100-11.

4. Khanna P, Paidas CN, Gilbert-Barness E. Alveolar soft part sarcoma: clinical, histopathological, molecular, and ultrastructural aspects. Fetal Pediatr Pathol. 2008:27:31-40

5. Ogose A, Yazawa Y, Ueda T, Hotta T, Kawashima H, Hatano H, Morita T. Alveolar soft part sarcoma in Japan: multi-institutional study of 57 patients from the Japanese Musculoskeletal Oncology Group. Oncology. 2003:65:7-13.

6. Anderson ME, Hornicek FJ, Gebhardt MC, Raskin KA, Mankin HJ. Alveolar soft part sarcoma: a rare and enigmatic entity. Clin Orthop Relat Res. 2005:438:144-8.

7. Lillehei KO, Kleinschmidt-DeMasters B, Mitchell DH, Spector E, Kruse CA. Alveolar soft part sarcoma: an unusually long interval between presentation and brain metastasis. Hum Pathol. 1993;24:1030-4.

8. Sood S, Baheti AD, Shinagare AB, Jagannathan JP, Hornick JL, Ramaiya NH, Tirumani $\mathrm{SH}$. Imaging features of primary and metastatic alveolar soft part sarcoma: single institute experience in 25 patients. Br J Radiol. 2014;87:20130719.

9. Zadnik PL, Yurter A, DeLeon R, Molina CA, Groves ML, McCarthy E, Sciubba DM. Alveolar soft-part sarcoma in the sacrum: a case report and review of the literature. Skelet Radiol. 2014:43:115-20.

10. Furey JG, Barrett DL, Seibert RH. Alveolar soft-part sarcoma. Report of a case presenting as a sacral bone tumor. J Bone Joint Surg Am. 1969:51:185-90.

11. Luzzati AD, Shah SP, Gagliano FS, Perrucchini GG, Fontanella W, Alloisio M. Four- and five- level en bloc spondylectomy for malignant spinal tumors. Spine. 2014;39:E129-139.
12. Zhu FP, Lu GM, Zhang LJ, Wang JD, An XJ, Dong YC. Primary alveolar soft part sarcoma of vertebra: a case report and literature review. Skelet Radiol. 2009;38:825-9.

13. Ordonez NG. Alveolar soft part sarcoma: a review and update. Adv Anat Pathol. 1999;6:125-39.

14. Ferrari A, Sultan I, Huang TT, Rodriguez-Galindo C, Shehadeh A, Meazza C, Ness KK, Casanova M, Spunt SL. Soft tissue sarcoma across the age spectrum: a population-based study from the surveillance epidemiology and end results database. Pediatr Blood Cancer. 2011:57:943-9.

15. McCarville MB, Muzzafar S, Kao SC, Coffin CM, Parham DM, Anderson JR, Spunt SL. Imaging features of alveolar soft-part sarcoma: a report from Children's Oncology Group Study ARST0332. AJR Am J Roentgenol. 2014; 203:1345-52.

16. Suh JS, Cho J, Lee SH, Shin KH, Yang Wl, Lee JH, Cho JH, Suh KJ, Lee YJ, Ryu KN. Alveolar soft part sarcoma: MR and angiographic findings. Skelet Radiol. 2000:29:680-9.

17. Selvarajah S, Pyne S, Chen E, Sompallae R, Ligon AH, Nielsen GP, Dranoff G, Stack E, Loda M, Flavin R. High-resolution array CGH and gene expression profiling of alveolar soft part sarcoma. Clin Cancer Res. 2014;20:1521-30.

18. Pradhan D, Roy S, Quiroga-Garza G, Cieply K, Mahaffey AL, Bastacky S, Dhir $\mathrm{R}$, Parwani AV. Validation and utilization of a TFE3 break-apart FISH assay for Xp11.2 translocation renal cell carcinoma and alveolar soft part sarcoma. Diagn Pathol. 2015;10:179.

19. Zarrin-Khameh N, Kaye KS. Alveolar soft part sarcoma. Arch Pathol Lab Med. 2007;131:488-91.

20. Persson S, Willems JS, Kindblom LG, Angervall L. Alveolar soft part sarcoma. An immunohistochemical, cytologic and electron-microscopic study and a quantitative DNA analysis. Virchows Arch A Pathol Anat Histol. 1988:412: 499-513.

21. Park YK, Unni KK, Kim YW, Han CS, Yang MH, Wenger DE, Sim FH, Lucas DR, Ryan JR, Nadim YA, et al. Primary alveolar soft part sarcoma of bone. Histopathology. 1999;35:411-7

22. Ladanyi M, Lui MY, Antonescu CR, Krause-Boehm A, Meindl A, Argani P, Healey JH, Ueda T, Yoshikawa H, Meloni-Ehrig A, et al. The der(17) t (X;17) (p11;q25) of human alveolar soft part sarcoma fuses the TFE3 transcription factor gene to ASPL, a novel gene at 17q25. Oncogene. 2001;20:48-57.

23. Reichardt $P$, Lindner $T$, Pink $D$, Thuss-Patience $P C$, Kretzschmar $A$, Dorken $B$. Chemotherapy in alveolar soft part sarcomas. What do we know? Eur J Cancer. 2003;39:1511-6.

24. Kayton ML, Meyers P, Wexler LH, Gerald WL, LaQuaglia MP. Clinical presentation, treatment, and outcome of alveolar soft part sarcoma in children, adolescents, and young adults. J Pediatr Surg. 2006:41:187-93.

25. Goldberg JM, Fisher DE, Demetri GD, Neuberg D, Allsop SA, Fonseca C, Nakazaki Y, Nemer D, Raut CP, George S, et al. Biologic activity of autologous, granulocyte-macrophage colony-stimulating factor secreting alveolar soft-part sarcoma and clear cell sarcoma vaccines. Clin Cancer Res. 2015;21:3178-86.

26. Judson I, Scurr M, Gardner K, Barquin E, Marotti M, Collins B, Young $H_{\text {, }}$ Jurgensmeier JM, Leahy M. Phase II study of cediranib in patients with advanced gastrointestinal stromal tumors or soft-tissue sarcoma. Clin Cancer Res. 2014;20:3603-12.

27. Stacchiotti S, Negri T, Zaffaroni N, Palassini E, Morosi C, Brich S, Conca E, Bozzi F, Cassinelli G, Gronchi A, et al. Sunitinib in advanced alveolar soft part sarcoma: evidence of a direct antitumor effect. Ann Oncol. 2011;22:1682-90.

28. Wagner AJ, Goldberg JM, Dubois SG, Choy E, Rosen L, Pappo A, Geller J Judson I, Hogg D, Senzer N, et al. Tivantinib (ARQ 197), a selective inhibitor of MET, in patients with microphthalmia transcription factor-associated tumors: results of a multicenter phase 2 trial. Cancer. 2012;118:5894-902.

29. Portera Jr CA, Ho V, Patel SR, Hunt KK, Feig BW, Respondek PM, Yasko AW Benjamin RS, Pollock RE, Pisters PW. Alveolar soft part sarcoma: clinical course and patterns of metastasis in 70 patients treated at a single institution. Cancer. 2001;91:585-91.

30. Lieberman PH, Brennan MF, Kimmel M, Erlandson RA, Garin-Chesa P, Flehinger BY. Alveolar soft-part sarcoma. A clinico-pathologic study of half a century. Cancer. 1989:63:1-13. 